\title{
Dynamic modeling of a solid oxide fuel cell system in Modelica
}

\author{
Daniel Andersson Erik Åberg Jonas Eborn \\ Modelon AB, Ideon Science Park, SE-223 70 Lund, Sweden \\ Jinliang Yuan Bengt Sundén \\ Department of Energy Science, Lund University, Box 118, SE-221 00
}

\begin{abstract}
In this study a dynamic model of a solid oxide fuel cell (SOFC) system has been developed. The work has been conducted in a cooperation between the Department of Energy Sciences, Lund University, and Modelon $\mathrm{AB}$ using the Modelica language and the Dymola modeling and simulation tool. The objective of the study is to investigate the suitability of the Modelica language for dynamic fuel cell system modeling.

Fuel cell system modeling requires a flexible modeling tool that can handle electronics, chemistry, thermodynamics and the interaction between these. The core of the fuel cell is the electrolyte and the electrodes. The cell voltage generated depends on the fluid molar compositions in the anode and cathode channels. The internal resistance varies depending on several cell properties. The electrical current through the cell varies over the cell area and is coupled to the rate of the chemical reactions taking place on the electrode surface. Other parts of the system that is also included in the model are pre-processing of the fuel, combustion of the fuel remaining after passing through the cell and heat recovery from the exhaust gas.
\end{abstract}

A cell electrolyte model including ohmic, activation and concentration irreversibilities is implemented and verified against simulations and experimental data presented in the open literature. A 1D solid oxide fuel cell model is created by integrating the electrolyte model and a 1D fuel flow model, which includes dynamic internal steam reforming of methane and water-gas shift reactions. Several cells are then placed with parallel flow paths and connected thermally and electrically in series. By introducing a manifold pressure drop, a stack model is created. This stack model is applied in a complete fuel cell system model including an autothermal reformer, a catalytic afterburner, a steam generator and heat exchangers. Four reactions are modeled in the autothermal reformer; two types of methane steam reforming, the water-gas shift reaction and total combustion of methane. Several simulations of systems and individual components have been performed, and when possible been compared with results in the literature. It can be concluded that the models are accurate and that Dymola and Modelica are tools well suited for simulations of the observed transient fuel cell system behaviour.

Keywords: SOFC; fuel cell; system model; dynamic reaction; reforming

\section{Nomenclature}

ASR Area specific resistance

C Cross-plane resistance area

D Diffusion coefficient

E Ohmic symmetry factor

F Faraday constant

G Gibbs free energy

$\mathrm{H} \quad$ Specific Enthalpy

$i \quad$ Current density

$i_{0} \quad$ Exchange current density

J Non-dimensional strip width

L Characteristic length

$n_{e} \quad$ Number of exchanged electrons

$p \quad$ Partial pressure

$\mathrm{P} \quad$ Pressure

R Universal gas constant

$r \quad$ Reaction rate

$t \quad$ Thickness

$\mathrm{T}$ Temperature

V Voltage

$\mathrm{X} \quad$ Cell pitch length

$y \quad$ Molar fraction 
$\alpha \quad$ Charge transfer coefficient

$\beta$ Parameter in Eq. (10)

$\gamma$ Pre-exponential factor

$\eta \quad$ Voltage loss

$v$ Effectiveness factor

$\rho$ Resistivity

$\sigma$ Conductivity

$\Omega \quad$ Denominator in reaction kinetics

\section{Introduction}

A solid oxide fuel cell is an electrochemical device that produces energy by oxidizing fuel from a replenishable source. A number of fuels are usable in SOFCs, thanks to internal reformation of the fuel. The overall reaction releasing energy is oxidation of hydrogen

$$
2 \mathrm{H}_{2}+\mathrm{O}_{2} \rightarrow 2 \mathrm{H}_{2} \mathrm{O}
$$

and thus the only byproduct produced aside from heat is water. The working principle is that oxygen is ionized at the cathode electrode by electrons from an external electrical circuit. The oxygen ions diffuse through the electrolyte to the anode electrode where they react with hydrogen gas, forming steam.

The solid oxide fuel cell is a high temperature fuel cell, most oftenly operating at $750-800^{\circ} \mathrm{C}$. The high temperature removes the need for expensive platinum catalyst and allows gases such as natural gas to be used as fuel directly. SOFCs are suitable for many types of system configurations. These include all sizes of combined heat and power applications, where a stationary fuel cell system is used to provide both electricity and heat to buildings, etc. The power generation in such systems can range from 2 kilowatts to several megawatts. They can also be used as auxiliary power units, or in hybrid systems where a fuel cell is combined with a gas turbine $[1,2,3]$. For all types of fuel cells it is important to keep track of the fuel composition going into the cell, as this affects the performance of the cell and in some cases can damage the cell. In this study a model of a SOFC system fueled with natural gas has been developed using Modelica and Dymola. Hydrogen is obtained from the natural gas via reformation. The fuel is reformed in an autothermal reformer prior to entering the cell, as well as during the flow through the cell channel; a process known as internal reforming. Heat is recovered from the exhaust gas and is used to generate steam from liquid water, as well as pre-heating the natural gas and air supplies.

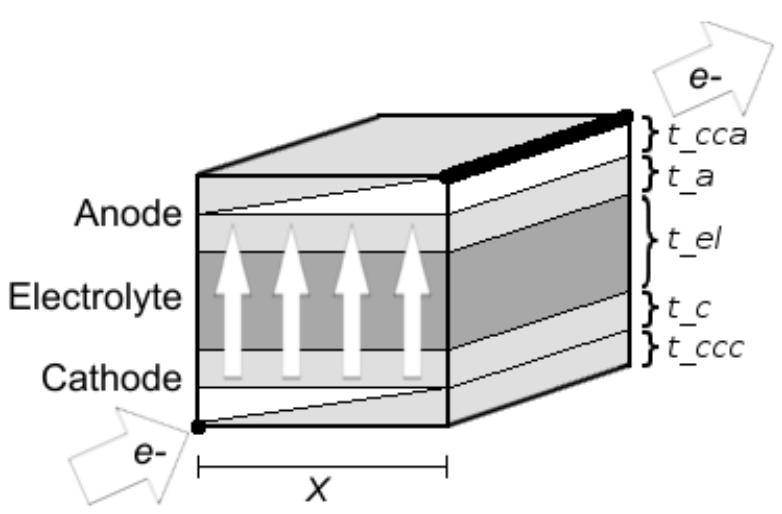

Figure 1: Geometry of the cell for calculation of the ohmic resistance

\section{Single cell model}

The ideal open circuit cell voltage is given by the Nernst equation [4]:

$$
V_{\text {Nernst }}=-\frac{\Delta G}{2 F}-\frac{R T}{2 F} \ln \left(\frac{p_{\mathrm{H}_{2} \mathrm{O}} p_{r e f}^{0.5}}{p_{\mathrm{H}_{2}} p_{\mathrm{O}_{2}}^{0.5}}\right)
$$

where $\Delta G$ is Gibbs free energy from the reaction and $p_{\text {ref }}$ is the standard pressure $0.1 \mathrm{MPa}$. When a current is applied, the cell voltage drops due to ohmic, activation and concentration losses, and thus the total voltage over the cell can be expressed as

$$
V=V_{\text {Nernst }}-\eta_{\text {Ohmic }}-\eta_{\text {act }}-\eta_{\text {conc }}
$$

\subsection{Ohmic loss model}

The calculation of the ohmic loss is based on the formulas for ohmic resistance of a cell with diagonal terminals, which is based on the integrated planar cell geometry as shown in Figure 1 [5]. For this geometry the area specific resistance is given by

$$
A S R=C J\left[\operatorname{coth}(J)+B\left(J-\tanh \left(\frac{1}{2} J\right)\right)\right]
$$

where $C$ is the cross-plane resistance area, given by:

$$
C=\rho_{c} t_{c}+\rho_{c c c} t_{c c c}+\rho_{e l} t_{e l}+\rho_{a} t_{a}+\rho_{c c a} t_{c c a}
$$

$B$ is given by

$$
\begin{aligned}
& B=\frac{E}{(1+E)^{2}} \\
& E=\left(\frac{t_{c c a}}{\rho_{c c a}}+\frac{t_{a}}{\rho_{a}}\right)^{-1}\left(\frac{t_{c c c}}{\rho_{c c c}}+\frac{t_{c}}{\rho_{c}}\right)
\end{aligned}
$$


where $E$ is the ohmic symmetry parameter. $J$ is given by

$$
\begin{aligned}
& J=\frac{X}{L} \\
& L=\sqrt{\frac{\rho_{e l} t_{e l}}{\left(\frac{t_{c c a}}{\rho_{c c a}}+\frac{t_{a}}{\rho_{a}}\right)^{-1}+\left(\frac{t_{c c c}}{\rho_{c c c}}+\frac{t_{c}}{\rho_{c}}\right)^{-1}}}
\end{aligned}
$$

where $X$ is the cell pitch length as indicated in Figure 1 and $L$ is the characteristic length [5]. The ohmic conductivities of the cell materials are temperature dependent, and commonly (e.g. [5]) calculated according to:

$$
\sigma=\rho^{-1}=\beta_{1} \exp \left(-\frac{\beta_{2}}{T}\right)
$$

The voltage drop is then calculated from the cell current density and the area specific resistance according to:

$$
\eta_{\text {Ohmic }}=A S R \cdot i
$$

\subsection{Activation loss model}

Activation losses occur at both the anode and cathode and derives from the fact that the reacting species in chemical reactions must overcome an energy barrier, i.e. the activation energy of the reaction. The activation loss shows a nonlinear current dependency related to a parameter known as the exchange current density, $i_{0}$. For current densities $i<i_{0}$ the activation loss is low, typically in the order of $0.01 \mathrm{~V}$, and for current densities $i>i_{0}$ the activation loss grows according to the Tafel equation:

$$
\eta_{a c t}=\frac{R T}{2 \alpha F} \ln \left(\frac{i}{i_{0}}\right)
$$

For current densities $i<i_{0}$ the activation loss is assumed to be proportional to the logarithm of the current density on the form:

$$
\eta_{\text {act }}=k \cdot \ln i
$$

Activation losses occur at both the anode and cathode sides which have different exchange current densities, denoted $i_{0, a}$ and $i_{0, c}$ respectively. These are calculated from the Arrhenius law and the composition of the reacting gases according to [5]:

$$
\begin{aligned}
& i_{0, c}=\gamma_{c}\left(\frac{p_{\mathrm{O}_{2}}}{p_{\text {ref }}}\right)^{0.25} \exp \left(-\frac{E_{a c t, c}}{R T}\right) \\
& i_{0, a}=\gamma_{a}\left(\frac{p_{\mathrm{H}_{2}}}{p_{\text {ref }}}\right)\left(\frac{p_{\mathrm{H}_{2} \mathrm{O}}}{p_{\text {ref }}}\right) \exp \left(-\frac{E_{a c t, a}}{R T}\right)
\end{aligned}
$$

\subsection{Concentration loss model}

Concentration losses accounts for the fact that when reactions occur, reactants and products must diffuse from the bulk flow to the reaction sites (and vice versa), through the porous electrodes. Because of this the actual pressure of the reactants and products differ from those in the bulk flow, and the voltage over the cell decreases. Typically this voltage drop is very low until the current density reaches a limiting current. Above this limit the concentration loss will have severe impact on cell performance and life time. The voltage drop is expressed as a function of the molar fractions $y_{i}$ of the gases at the reaction sites as [5]:

$$
\eta_{\text {conc }}=-\frac{R T}{n_{e} F}\left[\ln \left(\frac{y_{\mathrm{H}_{2}}^{*} y_{\mathrm{H}_{2} \mathrm{O}}^{O}}{y_{\mathrm{H}_{2}}^{O} y_{\mathrm{H}_{2} \mathrm{O}}^{*}}\right)+\frac{1}{2} \ln \left(\frac{y_{\mathrm{O}_{2}}^{*}}{y_{\mathrm{O}_{2}}^{O}}\right)\right]
$$

where $y^{*}$ indicate molar fraction at the reaction site, and $y^{O}$ indicate molar fraction in the bulk flow. The molar fractions at the reaction sites are calculated from those in the bulk, the current density through the electrolyte, the thickness of the anode and cathode, the bulk pressure and the diffusion coefficients [5], according to:

$$
\begin{aligned}
y_{O_{2}}^{*} & =1+\left(y_{O_{2}}^{O}-1\right) \exp \left(\frac{i R T t_{c}}{4 n_{e} F D_{O_{2}} P}\right) \\
y_{H_{2}}^{*} & =y_{H_{2}}^{O}-\frac{i R T t_{a}}{2 n_{e} F D_{H_{2}} P} \\
y_{H_{2} O}^{*} & =y_{H_{2} O}^{O}+\frac{i R T t_{a}}{2 n_{e} F D_{H_{2} O} P}
\end{aligned}
$$

\subsection{Simplified cell model}

In addition to the complete polarization model, modelling the internal losses physically as described above, also a simplified cell model was developed. In this model the internal losses are approximated by the following empirical correlation for area specific resistance [4]:

$$
A S R(T)=A S R_{0} \exp \left(\frac{E_{a}}{R}\left(\frac{1}{T}-\frac{1}{T_{0}}\right)\right)
$$

where the constant $A S R_{0}$ is the area specific resistance at temperature $T_{0}$. This simple model is not as accurate as the complete model, but gives shorter simulation times.

\subsection{Implementation}

The cell models are based on a template model containing common parts in all cell models. This includes 
connectors for mass flow, heat transfer and electrical current. Also a temperature state is introduced and energy balance is defined. The fuel and air compositions have large variations along the flow path due to reactions. To take this into account the cell is discretized in the fuel flow direction. The cell model is prepared for this by vectorizing all connectors and states that vary along the flow path. There is no heat transfer directly between the temperature states, this is covered by heat transfer to the surrounding wall and fluids, which also have heat transfer to the surrounding temperature states. The cell current is also discretized in the same way. For each element the current is coupled to the mass flow rate, and thereby the reaction rate at the electrodes, according to Faraday's laws of electrolysis.

\section{Substack model}

The cell model is connected to flow channel models in what we call a substack model. The flow channel models are volume models, discretized in flow direction. Every discrete element has a unique fluid composition and energy content. The anode channel include reactions for steam reforming of methane (21) and water-gas shift (22).

$$
\begin{aligned}
\mathrm{CH}_{4}+\mathrm{H}_{2} \mathrm{O} & \leftrightarrow \mathrm{CO}+3 \mathrm{H}_{2} \\
\mathrm{CO}+\mathrm{H}_{2} \mathrm{O} & \leftrightarrow \mathrm{H}_{2}+\mathrm{CO}_{2}
\end{aligned}
$$

In the channel model these reactions are characterized by a reaction object which contains the stoichiometry matrix and calculates the equilibrium constants of the reactions. The equilibrium constant is calculated from Gibbs free energy of the reaction, which is evaluated from the medium model, and the partial pressures of the reactants. The actual reaction rate is then calculated in the channel model from the deviation of the fluid composition from the calculated equilibrium. Medium properties are calculated by medium models from Modelon's CombiPlant library which is a model library for combined cycle power plant simulation. These medium models are compatible with the ones in the Modelica Standard Library, but also includes a model ReactionProperties which calculates properties related to reactions, such as Gibb's free energy due to reaction and reaction constants.

The two channel models are connected to a cell model according to Figure 2. The number of discrete elements are equal in the channel models and the cell

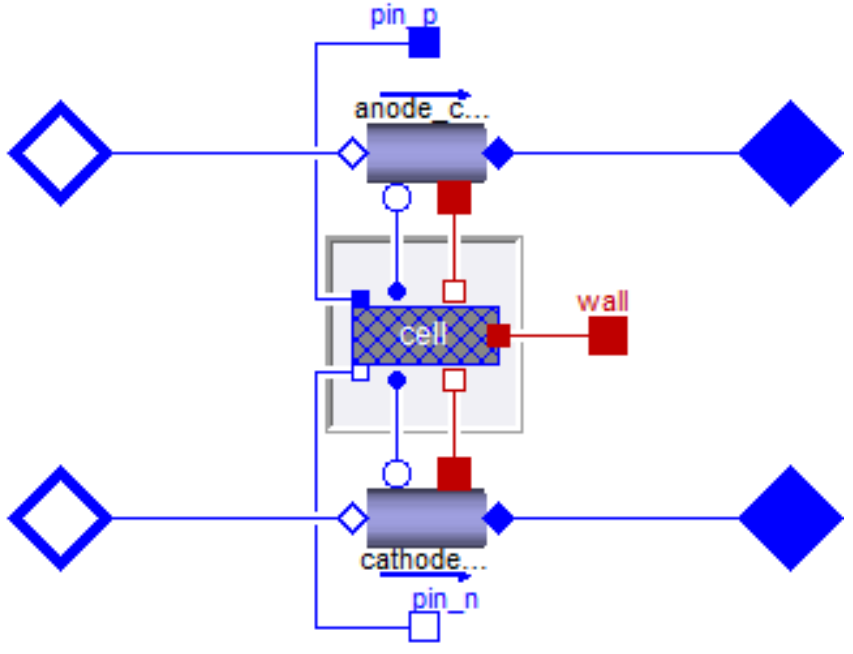

Figure 2: Graphical layout of the substack model

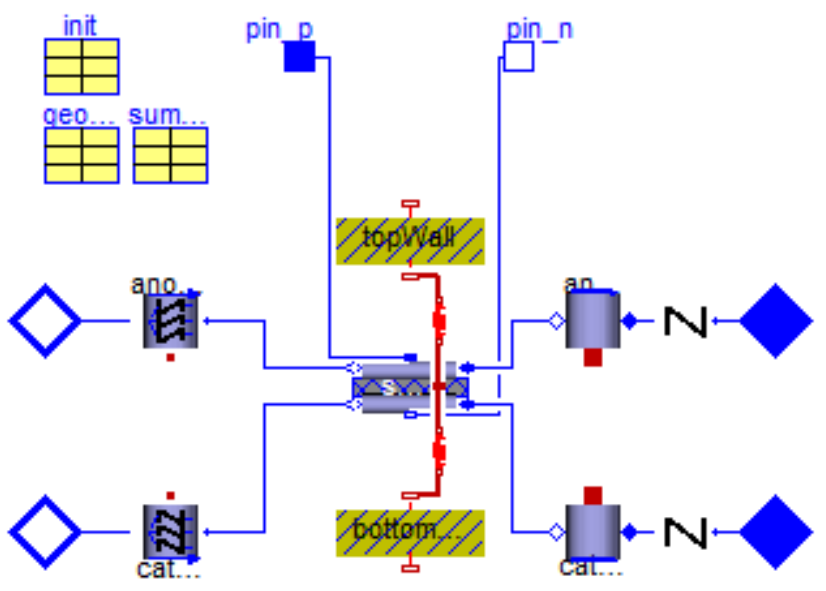

Figure 3: Graphical layout of the stack model

model. Thus the connections for mass and heat transfer are done per element, and each element in the cell model has a unique fuel and air state to evaluate. The substack models any number of cells by multiplying the affected quantities with the number of cells. The purpose of the substack model is to model a number of cells in the stack which can be assumed to have the same operating conditions.

\section{Stack model}

The complete stack is modeled by connecting several substack models, electrically and thermally in series, as shown in Figure 3. This model consists of a vector of substack models, inlet manifold volumes with pressure drop, and outlet manifold volumes. The first and last substacks in the vector are also thermally connected to thermal masses, modeling the heat capacity of the metal casing. The pressure drops in the in- 


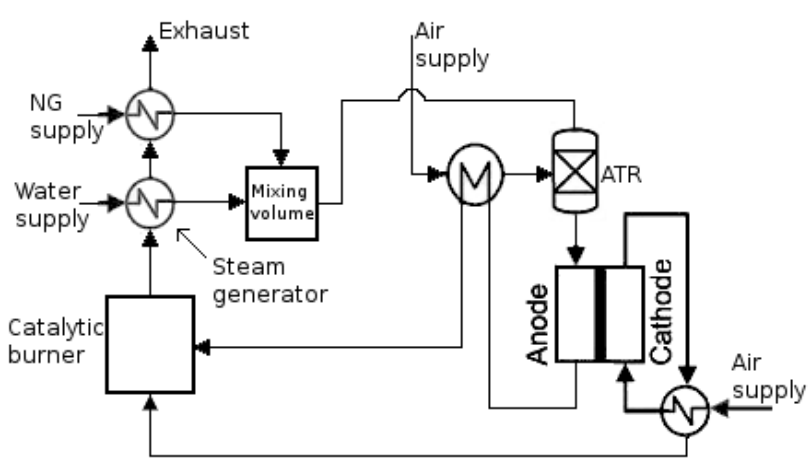

Figure 4: Layout of the implemented fuel cell system

let manifold volumes generates a unique pressure at each substack inlet, corresponding to a U-type manifold form. The stack model thereby has 3D effects from the heat boundary conditions and flow distribution.

\section{System model}

The system configuration is based on that presented in [6], with some modifications. The system layout, presented in Figure 4, includes an autothermal reformer (ATR), a steam generator and a catalytic burner. The outlet gases from the stack are fed through heat exchangers in order to pre-heat air and is then fed to a catalytic burner. The hot burner exhaust gas is used to vaporize liquid water to steam, which is needed for the reforming reactions in the ATR and stack and to pre-heat the natural gas from ambient temperature.

\subsection{ATR unit}

A large number of reactions can occur in an ATR unit, but in order to reduce the number of calculations only the most significant reactions are considered; steam reforming (23), (24), the water-gas shift reaction (25) and total combustion of methane (26) [7]:

$$
\begin{gathered}
\mathrm{CH}_{4}+\mathrm{H}_{2} \mathrm{O} \leftrightarrow \mathrm{CO}+3 \mathrm{H}_{2}, \\
\Delta H=206.2 \mathrm{~kJ} / \mathrm{mol} \\
\mathrm{CH}_{4}+2 \mathrm{H}_{2} \mathrm{O} \leftrightarrow \mathrm{CO}_{2}+4 \mathrm{H}_{2}, \\
\Delta H=164.9 \mathrm{~kJ} / \mathrm{mol} \\
\mathrm{CO}+\mathrm{H}_{2} \mathrm{O} \leftrightarrow \mathrm{CO}_{2}+\mathrm{H}_{2}, \\
\Delta H=-41.1 \mathrm{~kJ} / \mathrm{mol} \\
\mathrm{CH}_{4}+2 \mathrm{O}_{2} \leftrightarrow \mathrm{CO}_{2}+2 \mathrm{H}_{2} \mathrm{O}, \\
\Delta \mathrm{H}=-802.7 \mathrm{~kJ} / \mathrm{mol}
\end{gathered}
$$

The presented values for $\Delta H$ of the reactions are valid at $298 \mathrm{~K}$. These values are not used explicitly in the model, but are presented to show the mix of endothermic and exothermic reactions taking place in the ATR unit, allowing it to operate without any need for external heating or cooling systems. In our model enthalpies are evaluated from the medium model and depends on the thermodynamic state and composition of the gas in the ATR unit. The model is implemented as a volume model with dynamic reactions. The reaction rates are calculated as [7]:

$$
\begin{aligned}
& R_{1}=\frac{k_{1}}{p_{H_{2}}^{2.5}}\left(p_{C_{H_{4}}} p_{\mathrm{H}_{2} \mathrm{O}}-\frac{p_{\mathrm{H}_{2}}^{3} p_{C O}}{K_{I}}\right) \times \frac{1}{\Omega^{2}} \\
& R_{2}=\frac{k_{2}}{p_{H_{2}}^{3.5}}\left(p_{C_{4}} p_{H_{2} O}^{2}-\frac{p_{H_{2}}^{4} p_{C O_{2}}}{K_{I I}}\right) \times \frac{1}{\Omega^{2}} \\
& R_{3}=\frac{k_{3}}{p_{H_{2}}}\left(p_{C O} p_{\mathrm{H}_{2} \mathrm{O}}-\frac{p_{\mathrm{H}_{2}} p_{\mathrm{CO}_{2}}}{K_{I I I}}\right) \times \frac{1}{\Omega^{2}} \\
& R_{4}=\frac{k_{4 a} p_{C_{4}} p_{O_{2}}}{\left(1+K_{C_{4}}^{C} p_{C_{4}}+K_{O_{2}}^{C} p_{O_{2}}\right)^{2}}+ \\
& \frac{k_{4 b} p_{\mathrm{CH}_{4}} p_{\mathrm{O}_{2}}}{1+K_{\mathrm{CH}_{4}}^{C} p_{\mathrm{CH}_{4}}+K_{\mathrm{O}_{2}}^{C} p_{\mathrm{O}_{2}}} \\
& \Omega=1+K_{C O} p_{C O}+K_{H_{2}} p_{H_{2}}+ \\
& K_{\mathrm{CH}_{4}} p_{\mathrm{CH}_{4}}+K_{\mathrm{H}_{2} \mathrm{O}} \frac{p_{\mathrm{H}_{2} \mathrm{O}}}{P_{\mathrm{H}_{2}}}
\end{aligned}
$$

Here $R_{1}, R_{2}, R_{3}$ and $R_{4}$ are the corresponding reaction rates to reactions (23), (24), (25) and (26), respectively. $k_{j}$ is the Arrhenius reaction constant for reactions $j=1, \ldots, 4$ and is calculated using parameters from literature [7]. $K_{j}$ is the equilibrium constant for reaction $j$ and is listed in Table 1. The Van't Hoff species adsorption constants $K_{i}$ and $K_{i}^{C}$ for species $i$ are calculated in a similar way according to [7]:

$$
\begin{aligned}
K_{i} & =K_{o i} \times \exp \left(\frac{-\triangle H_{i}}{R T}\right) \\
K_{i}^{C} & =K_{o i}^{C} \times \exp \left(\frac{-\triangle H_{i}^{C}}{R T}\right)
\end{aligned}
$$

$$
\begin{array}{lc}
\text { Reaction, } j & \text { Equilibrium constant }, K_{j} \\
\hline 1 & K_{I}=\exp \left(\frac{-26830}{T_{s}}+30.114\right)\left[\mathrm{bar}^{2}\right] \\
2 & K_{I I}=K_{I} \cdot K_{I I I}\left[\mathrm{bar}^{2}\right] \\
3 & K_{I I I}=\exp \left(\frac{4400}{T_{s}}-4.036\right)\left[\mathrm{bar}^{2}\right]
\end{array}
$$

Table 1: Reaction equilibrium constants 
The determined reaction rates are used for calculating the rate of formation of each substance. This is done according to [7]:

$$
\begin{aligned}
r_{C_{4}} & =-v_{1} R_{1}-v_{2} R_{2}-v_{4} R_{4} \\
r_{O_{2}} & =-2 v_{4} R_{4} \\
r_{C O_{2}} & =v_{2} R_{2}+v_{3} R_{3}+v_{4} R_{4} \\
r_{H_{2} O} & =-v_{1} R_{1}-2 v_{2} R_{2}-v_{3} R_{3}-2 v_{4} R_{4} \\
r_{H_{2}} & =3 v_{1} R_{1}+4 v_{2} R_{2}+v_{3} R_{3} \\
r_{C O} & =v_{1} R_{1}-v_{3} R_{3}
\end{aligned}
$$

The $v$ factors are effectiveness factors used to account for the intraparticle transport limitation. Their values are set to $v_{1}=0.07, v_{2}=0.06, v_{3}=0.7$ and $v_{4}=0.05$, which are average values obtained from simulations of the various species inside the $\mathrm{Ni}$ catalyst pellet [8]. The low values for all reactions except the water gas shift reflect the severe limitation on the catalytic conversion of methane to synthesis gas due to intraparticle diffusion in the catalyst pellets. Calculation of effectiveness factors using various methods and a comparison of their effect on simulation results is presented in [9].

\subsection{Catalytic burner}

The catalytic burner model includes reactions for oxidation of methane (40), carbon monoxide (41) and hydrogen (42) [4]:

$$
\begin{aligned}
\mathrm{CH}_{4}+2 \mathrm{O}_{2} & \rightarrow \mathrm{CO}_{2}+2 \mathrm{H}_{2} \mathrm{O} \\
\mathrm{CO}+\frac{1}{2} \mathrm{O}_{2} & \rightarrow \mathrm{CO}_{2} \\
\mathrm{H}_{2}+\frac{1}{2} \mathrm{O}_{2} & \rightarrow \mathrm{H}_{2} \mathrm{O}
\end{aligned}
$$

The implementation of the burner is based on a volume model with air and fuel inlet ports and an air outlet port. It also has a port for heat flow to the environment and a boolean input for ignition signals. The burner operates in one of two states, either burning or mixing, where the difference is the stoichiometry matrix defining how the fuel components is mixed into the air flow. The burner can be ignited if the air/fuel ratio is low enough and the ignition signal is true. The burner is set to mixing state if the air/fuel ratio gets too high.

When combustion occurs a combustion rate defined as the fraction of fuel burned is calculated dynamically.

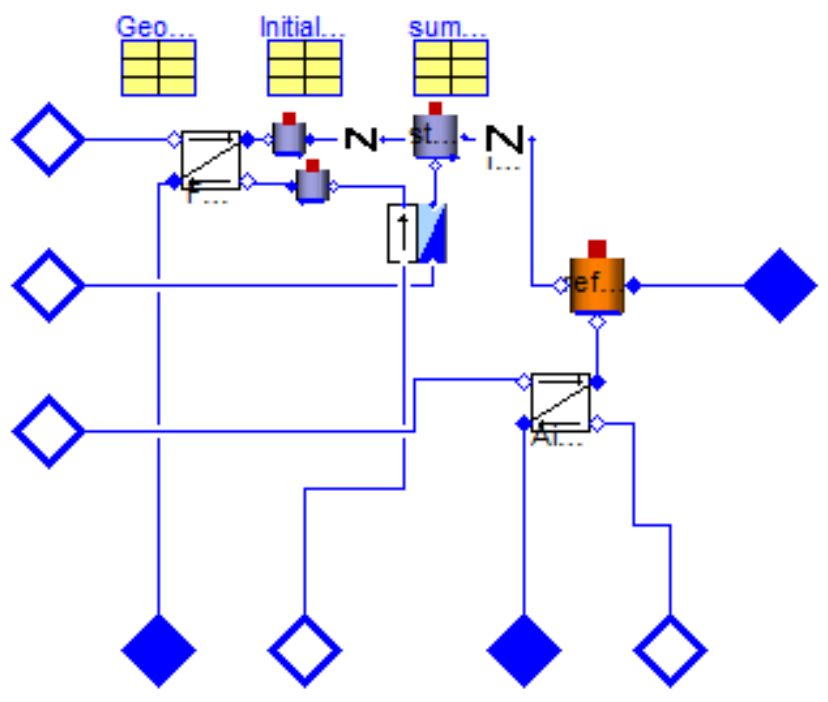

Figure 5: Graphical layout of the hotbox

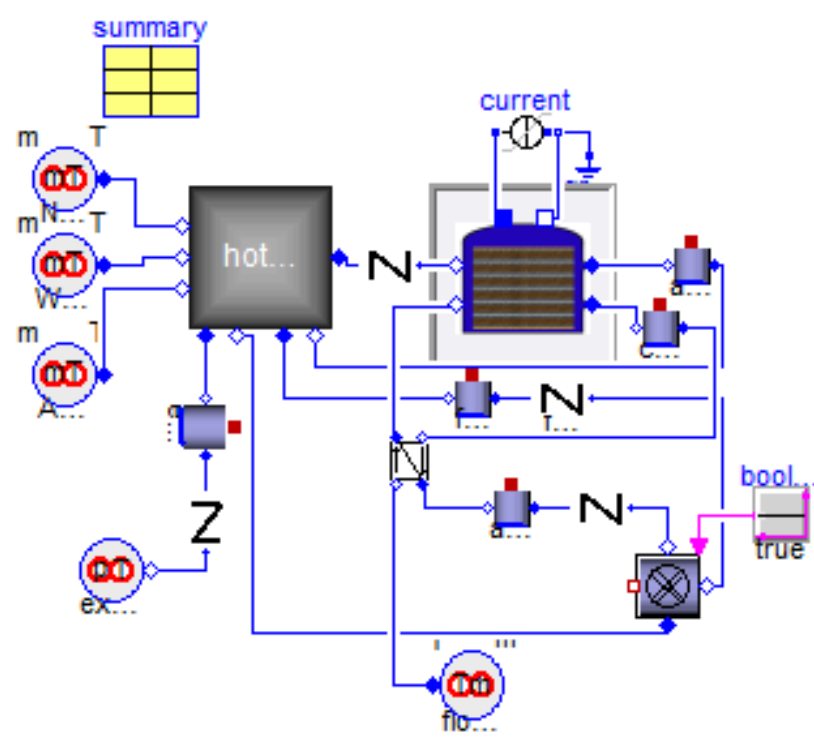

Figure 6: Graphical layout of the system model

\subsection{System implementation}

The system model is implemented in Dymola. Figure 5 shows a hotbox model, which includes the ATR unit, the steam generator, a mixing volume for natural gas and steam, and two heat exchangers for heat recirculation. In Figure 6 the complete system model is shown. It includes the hotbox and stack models, as well as the catalytic burner, a heat exchanger and flow sources of natural gas, water and air. Note that the implemented system uses co-flow of fuel and air in the stack, counter-flow can be obtained by reversing the air flow direction. 


\begin{tabular}{lr} 
Parameter & Value \\
\hline Anode thickness & $1 \mathrm{~mm}$ \\
Cathode thickness & $70 \mu \mathrm{m}$ \\
Electrolyte thickness & $10 \mu \mathrm{m}$ \\
Cell pitch length & $5 \mathrm{~mm}$ \\
Active cell area & $361 \mathrm{~cm}^{2}$ \\
Fuel molar fraction & $26.3 \% \mathrm{H}_{2}, 17.1 \% \mathrm{CH}_{4}$, \\
& $2.9 \% \mathrm{CO}, 4.4 \% \mathrm{CO}_{2}$, \\
Air oxygen fraction & $49.3 \% \mathrm{H}_{2} \mathrm{O}$ \\
& $23 \%$
\end{tabular}

Table 2: Cell geometry and fuel composition used in simulation

\section{Results}

Several simulations of the complete system and individual components are carried out in order to show their behaviour. Three of them are presented here. In all simulations the cells were discretized with four elements along the fuel flow direction. The effects of varying the number of elements has been investigated and four elements were found to give a good compromise between accuracy and computation time. When changing from 4 to 50 elements the resulting difference in cell voltage was $1.4 \%$, and the difference in total heat production was $1.8 \%$. If the purpose of the simulation is to locate hotspots or other local phenomena in the cell, more elements should be used. The cell geometry parameters and fuel composition used during the simulations are presented in Table 2. The values correspond to a $5 \mathrm{~kW}$ SOFC stack manufactured by Forschungszentrum Jülich, Germany, which is described in $[2,4]$.

\subsection{Substack with simplified cell model}

In this simulation the substack with the simplified cell model is simulated with a constant inlet flow rate of fuel and air. During the simulation the external current is increased linearly from 1 to $150 \mathrm{~A}$, which corresponds to a cell current density of 27.7 to $4155 \mathrm{~A} / \mathrm{m}^{2}$. The resulting cell voltage and power generation is shown together with simulation results from [4] in Figure 7. The reference model is a OD SOFC model where the Nernst potential is evaluated using an average fuel composition between the stack inlet and outlet. The marked upper and lower limits were obtained using the fuel composition at the stack inlet and outlet respectively. The difference between the results are not very large, but except for the different modeling approaches also different handling of heat contributes to
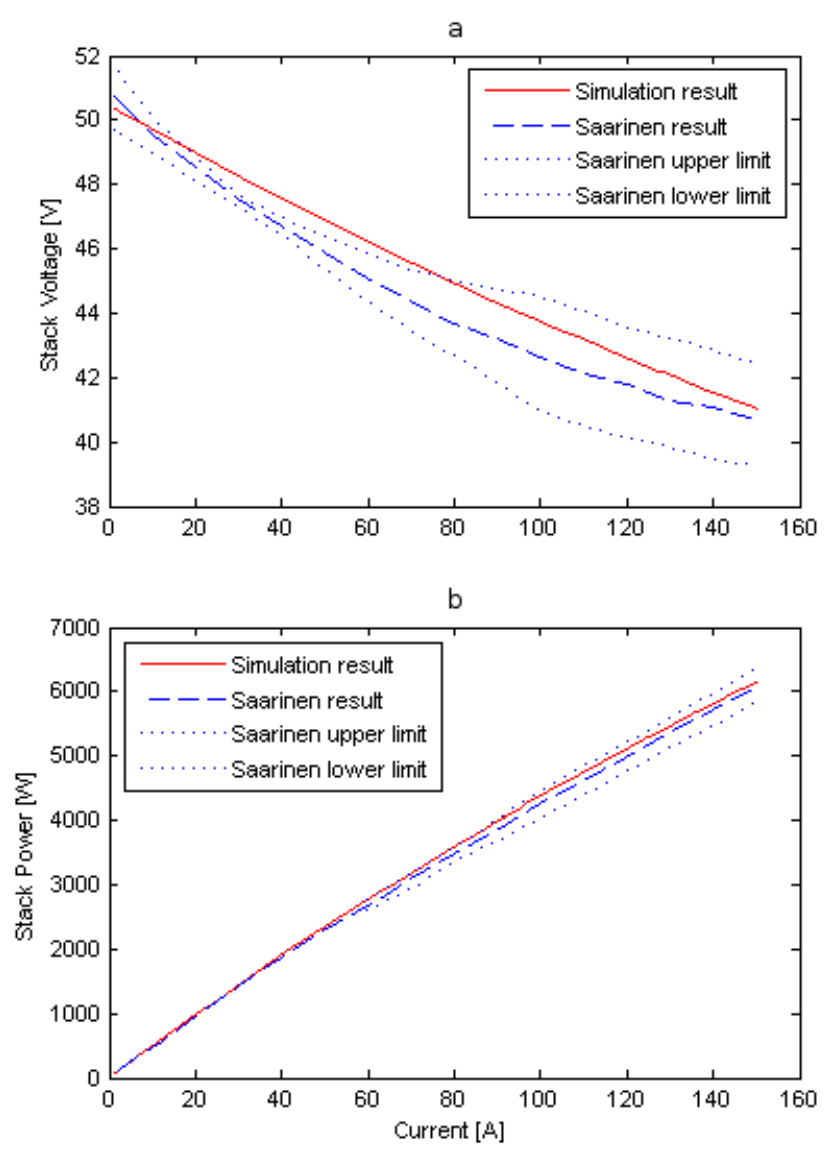

Figure 7: Simulation results for: (a) cell voltage, (b) cell power generation. Results from [4] is included for comparison.

the deviation. In the reference model the temperature was kept constant at $760^{\circ} \mathrm{C}$, whereas in our model the stack was cooled only by heat transfer between the gases and the inner surfaces of the fuel and air channels. This resulted in an increasing stack temperature with increased current, varying from $750^{\circ} \mathrm{C}$ to $810^{\circ} \mathrm{C}$.

\subsection{Substack with complete cell model}

In this simulation the substack with the complete cell model is simulated with a constant inlet flow rate of fuel and air. During the simulation the external current is increased linearly from 10 to 520 A, which corresponds to a cell current density of 277 to $14404 \mathrm{~A} / \mathrm{m}^{2}$ and fuel utilization of $30 \%$ to $100 \%$ for this specific cell.

In Figure 8(a) the cell voltage versus the cell current density shows the expected characteristics with a fast voltage drop for low current densities, a close to linear behaviour for medium current densities and a faster drop at high current densities. In Figure 8(b) the cell 


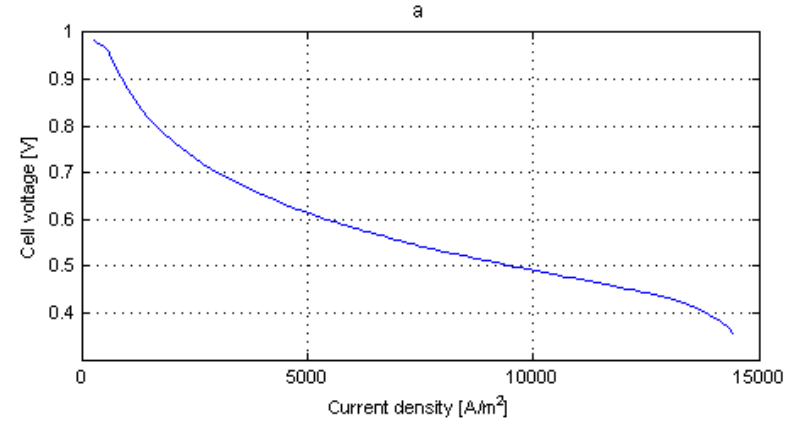

b

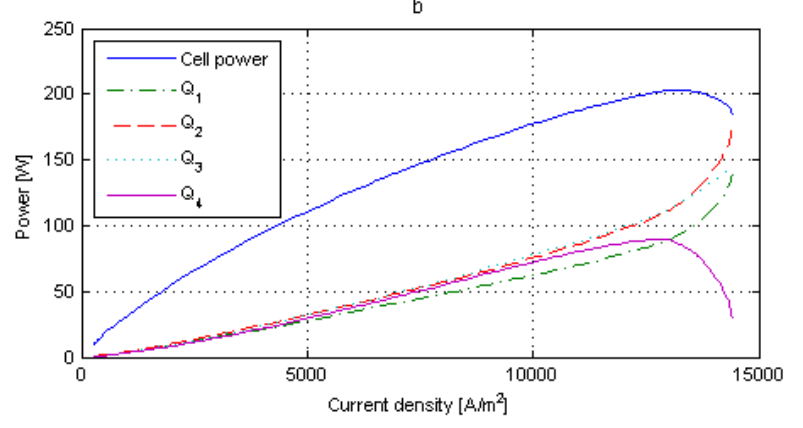

Figure 8: Simulation results for: (a) cell voltage, (b) cell power production and heat production in the four discrete elements

shows maximum power production at $13000 \mathrm{~A} / \mathrm{m}^{2}$. Also shown is the heat production in every discrete element, numbered from inlet to outlet. The heat production grows with increased current density and is roughly the same in each discrete element, except for very high currents when the heat production in the 4th element decreases rapidly. Figures 9(a)-(d) shows the fuel and air composition in every discrete element, numbered from inlet to outlet. The gradual consumption of hydrogen, methane and oxygen, and production of steam, can be observed.

\subsection{Complete system transient}

The system in Figure 6 is simulated with a constant external current of $150 \mathrm{~A}$, corresponding to a cell current density of $4155 \mathrm{~A} / \mathrm{m}^{2}$. Three substacks are included, the middle one modeling 30 cells, and the top and bottom ones modeling 10 cells each. The inlet flow rates to the hotbox are chosen such that a steam carbon ratio of 1.2 and a oxygen gas carbon ratio of 0.2 is achieved in the autothermal reformer, and the stack temperature is initialized at $800^{\circ} \mathrm{C}$. The simulation results for the system transient behaviour are presented in Figures 10 - 14. The substacks are numbered in the fuel flow direction of the manifold volume, i.e. substack 1 has the highest inlet pressure. In Figure 10 the middle substack
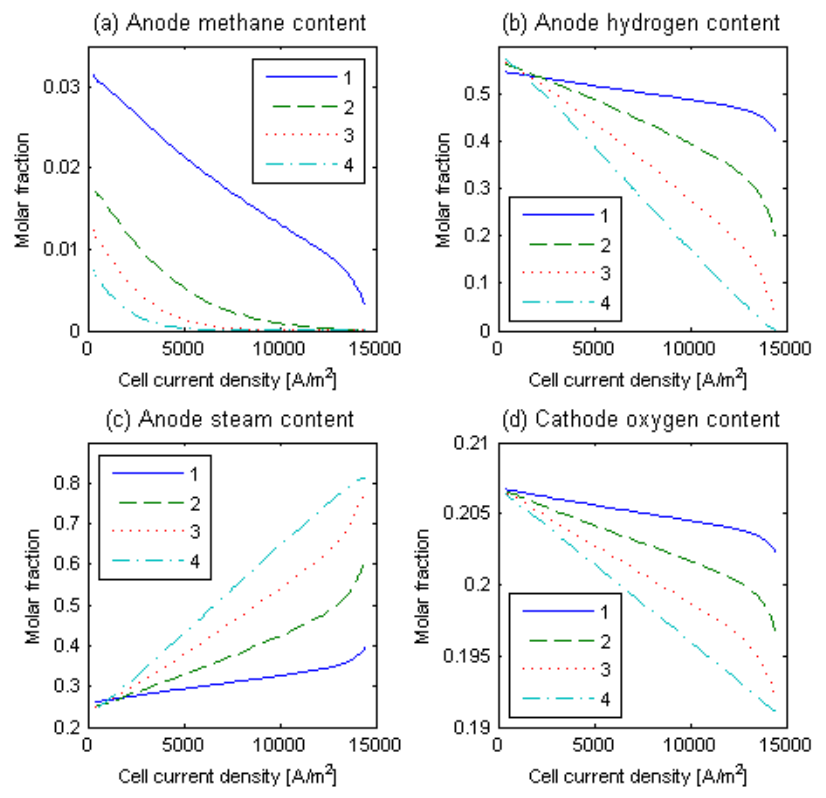

Figure 9: Simulation results for: (a) anode methane content, (b) anode hydrogen content, (c) anode steam content, (d) cathode oxygen content. Results shown for each discrete element, numbered from inlet to outlet.

shows the highest voltage per cell, due to the higher temperature than in the other substacks, as shown in Figure 11(a). The voltage losses in the cell decrease with increased temperature due to higher conductivity of the cell materials, and increased exchange current density which gives lower activation losses. The fuel utilization, shown in Figure 11(b), is highest in the lowermost substack, due to the pressure drop in the manifold volumes, which gives lower mass flow rates in the subsequent substacks. Presented in Figure 12 are the power and heat generation of the stack.

The resulting temperature profiles for the ATR unit and the catalytic burner are presented in Figure 13, together with the conversion ratios of methane and oxygen in the ATR. The oxygen conversion ratio is $1 \mathrm{ex}-$ cept for the the first seconds after start up. This is desirable, as oxygen in the stack inlet fuel flow decreases the overall system efficiency and destroys the stack as the Ni catalyst is oxidized. The high temperature of the burner is required for steam generation and pre-heating in the hotbox. The conversion ratio of methane in the ATR gives the stack inlet fuel composition presented in Figure 14. The composition reaches its steady-state value quickly, and then varies slightly with temperature. This composition complies with the results from similar simulations in [4]. 


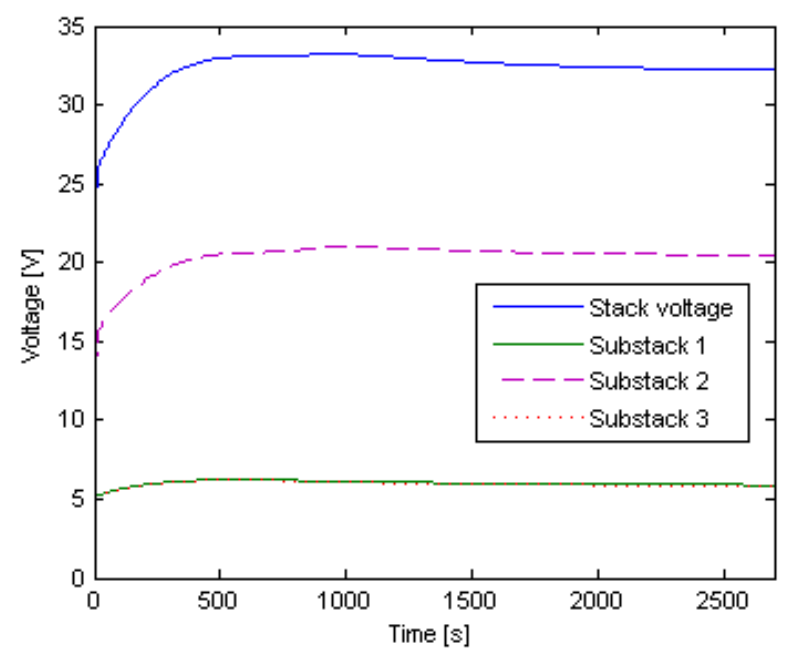

Figure 10: Dynamic simulation results for the voltage of the stack and individual substacks
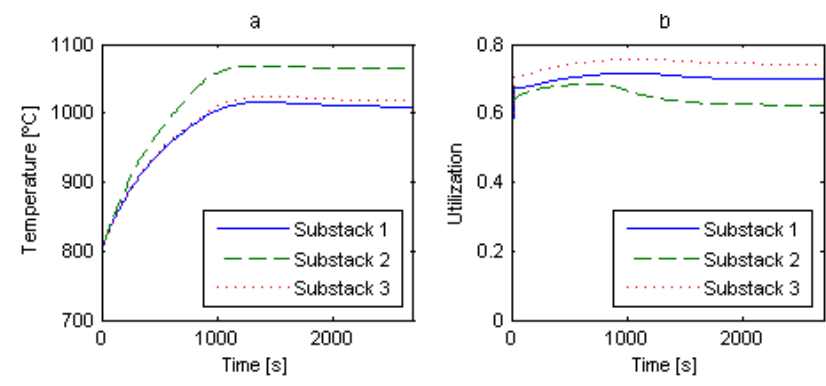

Figure 11: Dynamic simulation results for: (a) temperatures of the substacks, (b) hydrogen utilization of each substack

\section{Conclusions}

It is concluded that Dymola and Modelica are very useful tools for fuel cell system modeling. The positive aspects are mainly the equation based language, the multi domain possibilities, and the flexibility of an object oriented structure.

All developed components were simulated individually for verification. The comparison of the substack with the simplified cell model is presented in this paper. For the complete system it has not been possible to make relevant comparisons of absolute values, as geometry parameters and correlations for different components comes from different sources. The objective of this study was to assure that results obtained are phenomenologically sound. This was verified by running several simulations of the developed components under various boundary conditions and studying the influence on the results. The results of these simulations are presented in the original thesis [10].

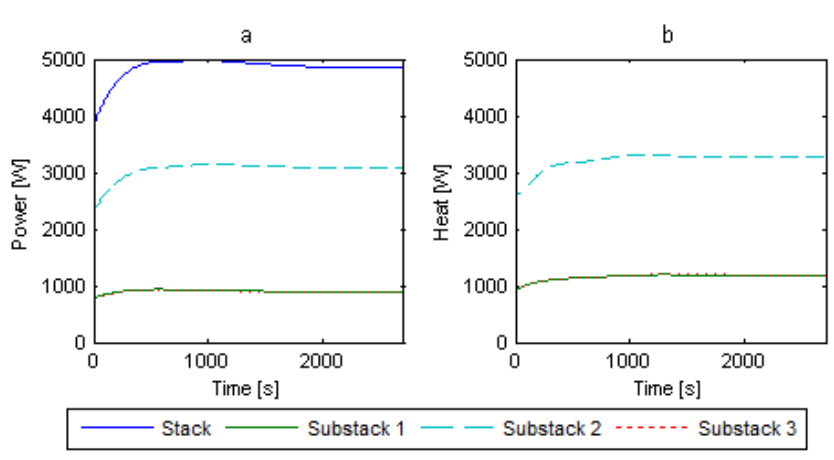

Figure 12: Dynamic simulation results for: (a) power generation of stack and individual substacks, (b) heat production of the substacks
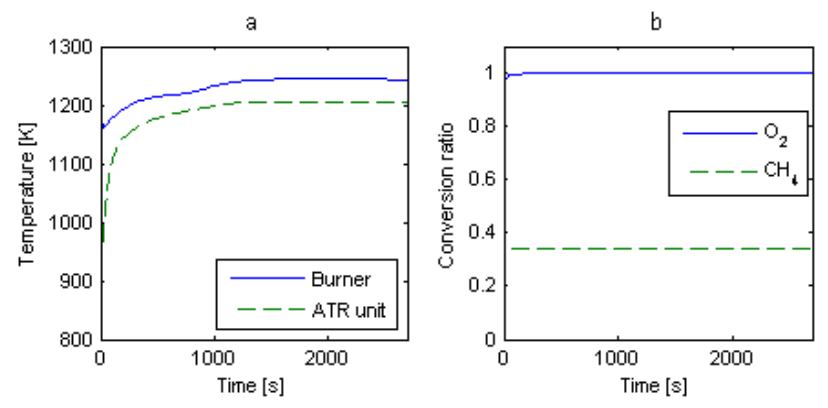

Figure 13: Dynamic simulation results for: (a) burner and ATR unit temperature, (b) ATR conversion ratio of methane and oxygen

As discussed in the abstract and introduction, modeling all relevant physics in a fuel cell system requires a flexible tool capable of including several physical domains in the same model. The equation based language and possibility for acausal models allows for physical models, which is very useful if components are to be reused in multiple system designs with different purposes. The system model developed in this study includes the electrochemistry of the cell, dynamic chemical reactions in the ATR unit as well as in the cell flow channel and the catalytic burner. It also includes heat exchanger models and a small electrical system. The system could easily be expanded to include a physical model of an electric load, or redesigned to another system configuration using the same component models.

One negative aspect can be mentioned. There is no automatic way to perform discretization of partial differential equations in Dymola, as some other nonModelica based tools provide. The discrete equations must be stated directly by the user which makes it harder and more time consuming than using tools de- 


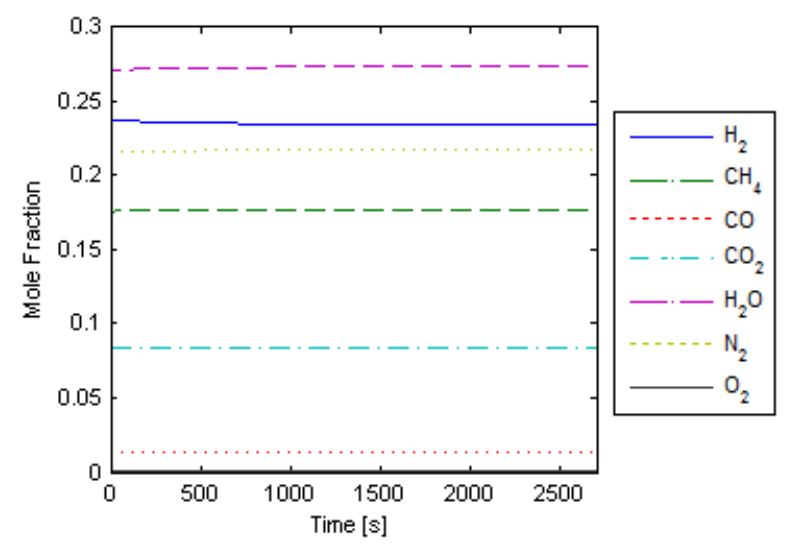

Figure 14: Dynamic simulation results for the ATR outlet fuel composition

veloped for this purpose. On the other hand this approach does not impose any limitations on how discretizations can be made and is thereby useable for many different applications.

\section{References}

[1] J. Larminie and A. Dicks. Fuel Cell Systems Explained, Second Edition. Wiley, 2003. ISBN 0470-84857-X.

[2] M. Kemm. Dynamic Solid Oxide Fuel Cell Modelling for Non-steady State Simulation of System Applications. PhD thesis, Division of Thermal Power Engineering, Lund University, 2006.

[3] S.H. Chan, H.K. Ho, and Y. Tian. Modelling of simple hybrid solid oxide fuel cell and gas turbine power plant. Journal of Power Sources, 109:111-120, 2002.

[4] J. Saarinen, M. Halinen, J. Ylijoki, M. Noponen, P. Simell, and J.Kiviaho. Dynamic model of 5 $\mathrm{kW}$ SOFC CHP test station. Journal of Fuel Cell Science and Technology, 4:397-405, 2007.

[5] P. Costamagna, A. Selimovic, M. Del Borghi, and G. Agnew. Electrochemical model of the integrated planar solid oxide fuel cell (IPSOFC). Chemical Engineering Journal, 102:6169, 2004.

[6] E. Fontell, T. Kivisaari, N. Christiansen, J.-B. Hansen, and J. Pålsson. Conceptual study of a $250 \mathrm{~kW}$ planar SOFC system for CHP application. Journal of Power Sources, 131:49-56, 2004.
[7] M.H. Halabi, M.H.J.M. de Croon, J. van der Schaaf, P.D. Cobden, and J.C. Schouten. Modeling and analysis of autothermal reforming of methane to hydrogen in a fixed bed reformer. Chemical Engineering Journal, 137:568-578, 2008.

[8] Ann M. De Groote and Gilbert F. Froment. Simulation of the catalytic partial oxidation of methane to synthesis gas. Applied Catalysis A: General, 138:245-264, 1996.

[9] Krzysztof Gosiewski, Ulrich Bartmann, Marek Moszczyński, and Leslaw Mleczko. Effect of the intraparticle mass transport limitations on temperature profiles and catalytic performance of the reverse-flow reactor for the partial oxidation of methane to synthesis gas. Chemical Engineering Science, 54:4589-4602, 1999.

[10] Daniel Andersson and Erik Åberg. Dynamic modeling of a solid oxide fuel cell system in Modelica. Master's thesis, Department of Energy Sciences, Lund University, 2010. 